\title{
EBM
}

\section{Leistungen vollständig erbringen und Berichtspflicht erfüllen}

\author{
"Eine Gebührenordnungsposition ist nur berechnungsfähig, wenn der \\ Leistungsinhalt vollständig erbracht worden ist." So steht es in den \\ Allgemeinen Bestimmungen 2.1. Und unter Punkt 2.1.2 heißt es dann: \\ "Eine Gebührenordnungsposition, deren Leistungsinhalt nicht vollstän- \\ dig erbracht wurde, kann nicht berechnet werden". Soweit ist alles klar. \\ Ein paar Besonderheiten gibt es aber doch.
}

$\mathrm{N}$ icht abgerechnet werden können Leistungen, die vorzeitig abgebrochen werden, wie beispielsweise eine Infusion, weil der Punktionsversuch nicht gelingt. Auch bei Nichterreichen einer vorgeschriebenen Mindestzeit, wie etwa beim Langzeit-EEG (GOP 16311, Ableitungsdauer mindestens zwei Stunden) ist diese Leistung nicht abrechnungsfähig. Die Gründe für die nicht komplette Erbringung der Leistung spielen dabei keine Rolle, auch wenn es am Patienten liegt, der vielleicht zu einem zweiten Termin nicht erschienen ist. Eine Privatliquidation einer solchen Teilleistung wäre nur dann möglich, wenn der Patient vorher auf diese Variante hingewiesen wurde.

Eine besondere Situation ist gegeben, wenn ein Hausbesuch bestellt wurde, der Patient aber nicht angetroffen wurde (z.B. zwischenzeitlicher Ruf des Notarztwagens und Transport in das Krankenhaus). In diesen Fällen kann zumindest der Besuch und das entsprechende Wegegeld abgerechnet werden, aber doch keine Grundpauschale oder sons-

tige Leistung (Beschluss Nr. 812 der Partner des Bundesmantelvertrages: „Wird ein Vertragsarzt in dringenden Fällen [z.B. zu einem Verkehrsunfall] gerufen und wird der Patient nicht angetroffen, so kann der Vertragsarzt unter Angabe von Gründen die GOP 01411 oder 01412 in voller Höhe berechnen.").

Werden Leistungen bei arztpraxisübergreifender Tätigkeit (Stamm- und Zweigpraxis) durch denselben Arzt erbracht, sind diese über die Betriebsstätte abzurechnen, wo die Leistungserfüllung beendet worden ist. Auch wenn in einer BAG mehrere Ärzte an der Erfüllung einer Leistung beteiligt sind, erfolgt die Abrechnung über die Arztnummer des Kollegen, der die Leistung letztendlich vollendet hat.

\section{Berichtspflicht}

Im Rahmen der Vollständigkeit der Leistungserbringung ist die „Berichtspflicht“ gesondert zu erwähnen. Grundsätzliche Angaben hierzu macht der Bundesmantelvertrag Ärzte und Krankenkassen (\$24 Abs. 6) und das SGB V im \$ 73. Ein-

Tab. 1: GOP mit Berichtspflicht für Neurologen und Psychiater

Leistungen, die für die Abrechnung einen Bericht oder Brief an den Hausarzt erfordern:

Leistungen, die bei Überweisung durch einen anderen Facharzt, eine Befundkopie an den Hausarzt verlangen: zelheiten finden sich aber auch in den Allgemeinen Bestimmungen des EBM, im Punkt 2.1.4. Im zweiten Absatz dieses Punktes sind die GOP aufgelistet, die nur dann vollständig erbracht sind und abgerechnet werden dürfen, wenn ein Bericht gemäß GOP 01600 oder ein Brief gemäß GOP 01601 an den Hausarzt geschickt wurde. Gleiches ist zu tun, wenn der Patient von einem anderen Facharzt überwiesen wurde. Die entsprechenden GOP für Neurologen und Psychiater sind in Tab. 1 aufgelistet.

In einem weiteren Absatz finden sich etliche fachärztliche Leistungen, deren vollständige Erbringung nur dann gewährleistet ist, wenn zumindest eine Befundkopie an den Hausarzt ging (Tab. 1).

Im Juli 2011 wurde $\$ 73$ Abs. 1b dahingehend ergänzt, dass für die Übersendung von Arztbriefen und Berichten die Einwilligung des Patienten vorliegen muss. Ist das nicht der Fall oder wird kein Hausarzt genannt, kann trotzdem abgerechnet werden. Jedoch müssen diese Ausnahmesituationen in der Dokumentation erkennbar sein. In einigen KVen müssen die Fälle mit einer Sondernummer versehen werden. Einzelheiten bei Ihrer KV.

Die Erstellung von Arztbrief oder Bericht kann mit den GOP 01600 beziehungsweise 01601 abgerechnet werden, soweit diese nicht obligater oder fakultativer Bestandteil der Leistung selbst ist. Für den Versand kann die GOP 40120 genutzt werden.

Dass zur Abrechnung einer Leistung diese vollständig erbracht werden muss, keine Frage. Für manchen lästig ist vielleicht die klar definierte Berichtspflicht. Die Ausnahmeregelung macht die Abrechenbarkeit da möglich, wo Patienten aus irgendwelchen Gründen der Übermittlung widersprechen.

Dr. med. Heiner Pasch 